\title{
Parameter Estimation of Short Duration Data Using Fast Adaptive Algorithm
}

\author{
${ }^{O}$ Hirofumi Yogo, Tadashi Kitamura and Naoki Inagaki \\ Department of Electrical and Computer Engineering \\ Nagoya Institute of Technology \\ Gokiso-cho, Showa-ku, Nagoya 466,Japan
}

\begin{abstract}
A fast and new parameter estimation method is proposed in this paper. The proposed method utilizes the inverse system based on an adaptive digital filter (ADF). As an adaptive algorithm, "Accelerated Stochastic Approximation (ASA) " method is used. The ASA method adopts the time-varying and the optimized convergence factor, and converges within 25 iterations. As the applications of the proposed method, two examples of the parameter estimation of the ARMA model and the natural stop consonants are presented.
\end{abstract}

\section{Introduction}

A method of an adaptive signal processing is utilized for a wide variety of applications.[1]-[6] The method is useful for a time-varying signal,specifically varying-signal in a short time. A new parameter estimation method of a short duration data based on adaptive technique is proposed in this paper. As an example of the short duration data, stop consonants were used. Stop consonants are composed of the consonant and the vowel segment, and their intrinsic features exist within the short duration (about $10 \mathrm{mS}$ to $20 \mathrm{mS}$ ) between the consonant and the vowel segment Speech production process of the stop consonant is expressed by an autoregressive and movingaverage (ARMA) model. Several intricated procedures have been utilized for the parameter estimation of the ARMA model.[7]-[8] This paper showed that a fast parameter estimation of the short duration data was facilitated by using the method based on an adaptive digital filter (ADF). As an adaptive algorithm of the $\mathrm{ADF}$, a fast adaptive algorithm was developed by improving a conventional stochastic approximation (SA) method [9]. The algorithm was named the Accelerated Stochastic Approximation (ASA) method.[10] The ASA method converges within 25 iterations. When a sampling frequency of 10
$\mathrm{kHz}$ is used, only $2.5 \mathrm{mS}$ duration data is needed for the parameter estimation.

As the applications of the proposed method, the parameter estimation of the ARMA model and the stop consonants was performed.

\section{Accelerated Stochastic Approximation (ASA) method}

The ASA method uses the time-varying and optimized convergence factor with respect to each parameter to be estimated, and is described in Eq.(1).

$W(j+1)=W(j)+e(j) P(j)^{T} \mu(j) /(j+h)$

where

$W(j)=\operatorname{col}\left[b_{0}(j), \cdots, b_{N}(j), a_{1}(j), \cdots, a_{M}(j)\right]$
$P(j)=\operatorname{col}\left[\beta_{0}(j), \cdots, \beta_{N}(j), \alpha_{1}(j), \cdots, \alpha_{M}(j)\right]$
$\mu(j)=\operatorname{diag}\left[\mu_{b 0}(j), \cdots, \mu_{b N}(j), \mu_{a 1}(j)\right.$,
$\left.\cdots, \mu_{a M}(j)\right]$

The $\alpha_{k}(j)$, and $\beta_{p}(j)$ are the gradient with respect to error $e(j)$, and are given in Eqs. (2) and (3). 
$\alpha_{k}(j)=-\partial e(j) / \partial a_{k}(j), \quad k=1, \cdots, M$

$\beta k(j)=-\partial e(j) / \partial b_{k}(j), \quad k=0, \cdots, N$

$\alpha_{k}$ and $\beta_{k}$ are given in Eqs. (4) and (5)

$$
\begin{array}{r}
\alpha_{k}(j)=y(j-k)+\sum_{i=1}^{M} a_{i}(j) \alpha_{k}(j-i) \\
k=1, \cdots, M
\end{array}
$$

$$
\begin{array}{r}
\beta_{k}(j)=x(j-k)+\sum_{i=1}^{N} a_{i}(j) \beta_{k}(j-i) \\
k=0, \cdots, N
\end{array}
$$

Moreover, $a_{k}(j)$ and $b_{k}(j)$ are calculated by Eqs. (6) and (7).

$$
a_{k}(j+1)=a_{k}(j)+u e(j) \alpha_{k}(j),
$$

$b_{k}(j+1)=b_{k}(j)+v e(j) \beta_{k}(j)$,

$e(j)=d(j)-y(j), \quad d(j)$ : disired value $y(j)$ : output of the ADF, T: transpose, $\mathrm{x}(\mathrm{j})$ : input of the $\mathrm{ADF}, \quad \mathrm{h}$ : constant, $\mathrm{j}$ : time index, u, v: constant

Individual convergence factors $\mu_{\mathrm{ak}}(\mathrm{j})$ and $\mu_{\mathrm{bp}}(\mathrm{j})$ are given in Eqs. (8) and (9).

$$
\begin{gathered}
\mu_{\mathrm{ak}}(\mathrm{j})=\mu_{0}\left(1-\mathrm{e}^{-\|e(j) x(j)\|}\right) /\left(1+\mathrm{e}^{\mathrm{nj}}\right) . \\
\mu_{\mathrm{ak}}{ }^{*}(\mathrm{j}) \quad, \mathrm{k}=1, \cdots \mathrm{M}
\end{gathered}
$$

The $\mu_{\mathrm{ak}} *(\mathrm{j})$ and $\mu_{\mathrm{bp}} *(\mathrm{j})$ denote the optimized individual convergence factors[11]-[12] and are given in Eqs.(10) and (11).

$$
\begin{array}{r}
\mu_{\mathrm{ak}} *(\mathrm{j})= \\
\underset{\mathrm{i}=1}{\mathrm{~N}}\left\{\partial \mathrm{\partial e}(\mathrm{j}) / \partial \mathrm{a}_{\mathrm{i}}(\mathrm{j})\right\}^{3}
\end{array}
$$

$$
\begin{gathered}
\mu_{\mathrm{bp}} *(\mathrm{j})= \\
\sum_{\mathrm{i}=1}^{\mathrm{M}}\left\{\partial \mathrm{de}(\mathrm{j}) / \partial \mathrm{b}_{\mathrm{i}}(\mathrm{j})\right\}^{3}
\end{gathered}
$$

where

$\alpha=\mathrm{M}+\mathrm{N}) /(\mathrm{M}+\mathrm{N}+1), \quad \mu_{0}=1 /(\mathrm{M}+1) \cdot \sigma^{2}$

\|\| : vector norm, $\sigma^{2}$ : input power,

$\mathrm{M}, \mathrm{N}$ : model order, $\mathrm{n}$ : constant

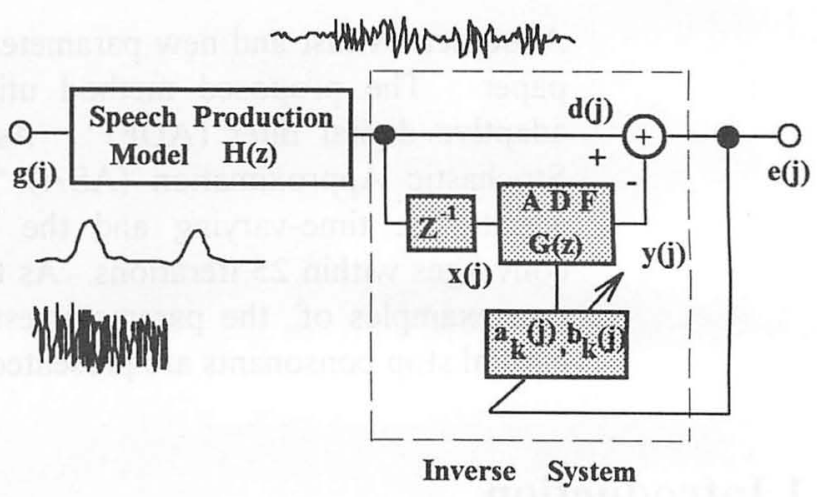

Fig.1 Block diagram of the inverse system using ADF.
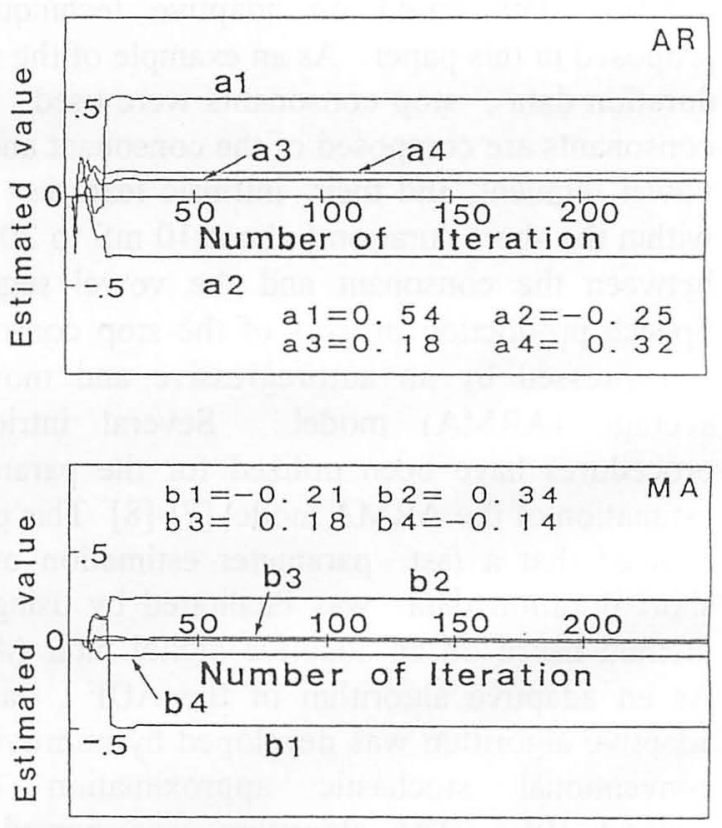

Fig.2 Convergence property of the inverse system. 


\section{Parameter estimation of the ARMA model}

The parameter estimation scheme of the ARMA model with known parameters is shown in Fig.1. $H(z)$ denotes a transfer function of the speech production model, and is given in Eq. (12).

$$
\begin{aligned}
\mathrm{H}(\mathrm{z})= & \left(1+\theta_{1} \mathrm{z}^{-1}+\cdots+\theta_{\mathrm{q}} \mathrm{z}^{-\mathrm{q}}\right) / \\
& \left(1 \cdots-\Phi_{1} \mathrm{z}^{-1}-\cdots-\Phi_{\mathrm{p}} \mathrm{z}^{-\mathrm{p}}\right)
\end{aligned}
$$

where

$\Phi_{1}, \cdots, \Phi_{\mathrm{p}}$ denote $\mathrm{AR}$ parameters, and $\theta_{1}, \ldots, \theta_{\mathrm{q}}$ ,MA parameters.

Moreover, $G(z)$ denotes the tranfer function of the ADF, and given in Eq. (13).

$$
\begin{aligned}
\mathrm{G}(\mathrm{z})= & \left(\mathrm{b}_{1}+\mathrm{b}_{2} \mathrm{z}^{-1}+\cdots+\mathrm{b}_{\left.\mathrm{p} \mathrm{z}^{-\mathrm{p}+1}\right) /}\right. \\
& \left(1-\mathrm{a}_{1} \mathrm{z}^{-1}-\cdots \cdot \mathrm{a}_{\mathrm{q}} \mathrm{z}^{-\mathrm{q}}\right)
\end{aligned}
$$

where

$a_{1}, \cdots, a_{q}$ denote AR parameters and $b_{1}, \cdots, b_{p}$ , MA parameters.

The parameters of the ARMA model are obtained by using the inverse system based on ASA method, and by minimizing the estimation errors. The estimated parameters are given in Eqs. (14) and (15).

$$
\begin{array}{ll}
\Phi_{\mathrm{k}}=\mathrm{a}_{\mathrm{k}}+\mathrm{b}_{\mathrm{k}} & \mathrm{k}=1, \cdots, \mathrm{p} \\
\theta_{\mathrm{k}}=-\mathrm{a}_{\mathrm{k}} & \mathrm{k}=1, \cdots, \mathrm{q}
\end{array}
$$

An example of the converegence characteristics of the ARMA $(4,4)$ model is shown in Fig.2. The AR and the MA parameters are converged less than 25 iterations.

In the next place, impulse responses of both the known and the estimated models are calculated for the comparison of the estimation accuracy. And then, 1024 points FFT tranformations of the impulse responses are performed, and the power spectra were obtained. The number of the data derived from the impulse response is small, and zero data was added. The procedure is shown in Fig.3, and the results are shown in Fig.4. Fig.4(a) shows the power spectra based on the estimated parameters, and Fig.4(b) the spectra based on the known parameters. The estimated spectra have a little degraded characteristics in zero at high frequency. However, we can recognize that the almost similar properties were obtained. Therefore, it is presumed that the reasonable parameter estimations based on the inverse system are performed.

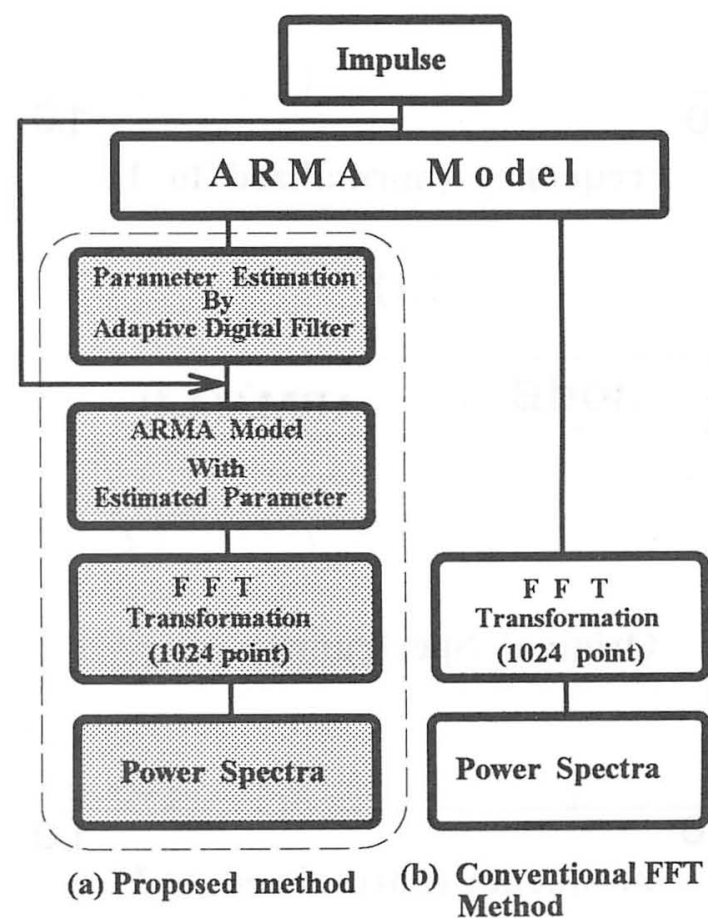

Fig.3 Block diagram of parameter estimation (ARMA model)

\section{Analysis of Stop Consonants}

As an application of the proposed method, feature extraction of the voiced and the unvoiced stop consonants was performed according to Fig. 5 . The $\mathrm{AR}$ and the MA parameters are estimated from the transitional portion between the consonant and the vowel segment without any paticular restrictions. The algorithm utilized converges within 25 iterations, and only needs 2.5 $\mathrm{mS}$ duration data. The model order was fixed to 6 because the degraded result was not obtained even though the model orders was selected to larger value than the presumed one. 


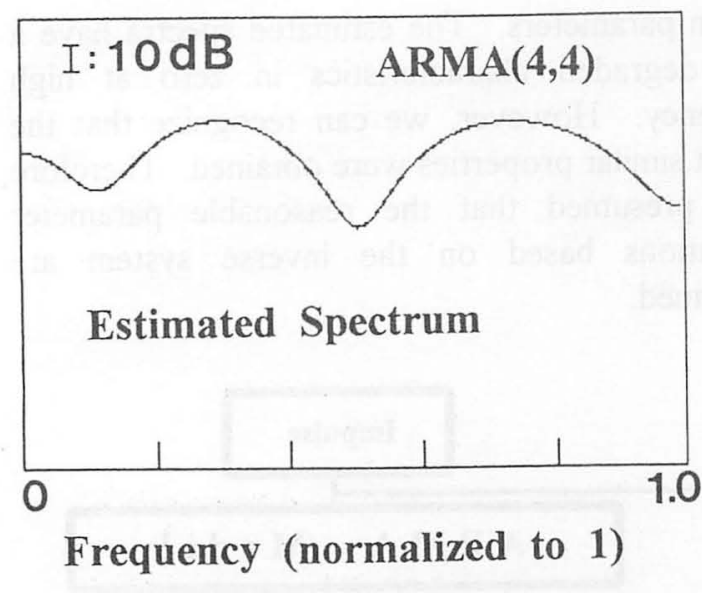

(a)

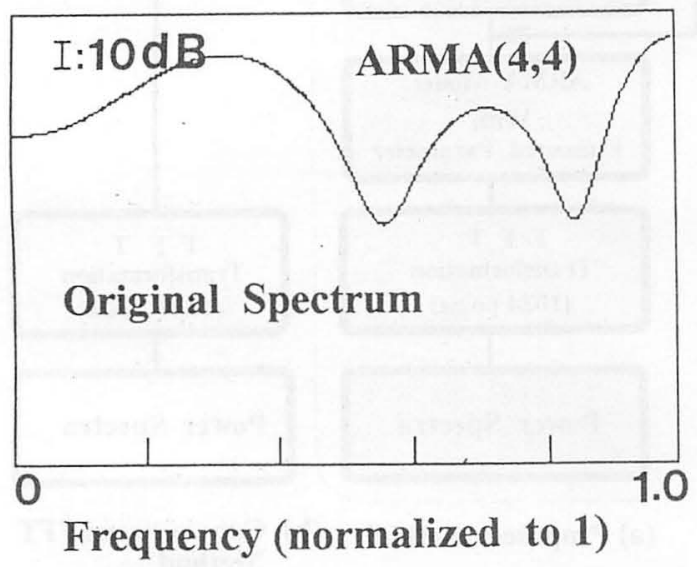

(b)

Fig.4 Estimated spectrum (ARMA model)

(a) Proposed method

(b) Conventional FFT method

In the following, the impulse responses of the ARMA model with the estimated parameters were calculated. And the power spectra were obtained by executing 1024 point FFT trasformation of the impulse response. The spectra of the unvoiced stop consonants are shown in Fig.6. The abovementioned procedures were repeated 11 times for the transitional portion by shifting $1 \mathrm{mS}$ with each frame of $2 \mathrm{mS}$. Fig.6(a) shows the results based on the proposed method, and Fig.6(b) the spectra obtained by a conventional FFT transformation of 25 point real speech data. Moreover, the parameter estimation and the spectra calculation of the voiced stop consonants were performed using

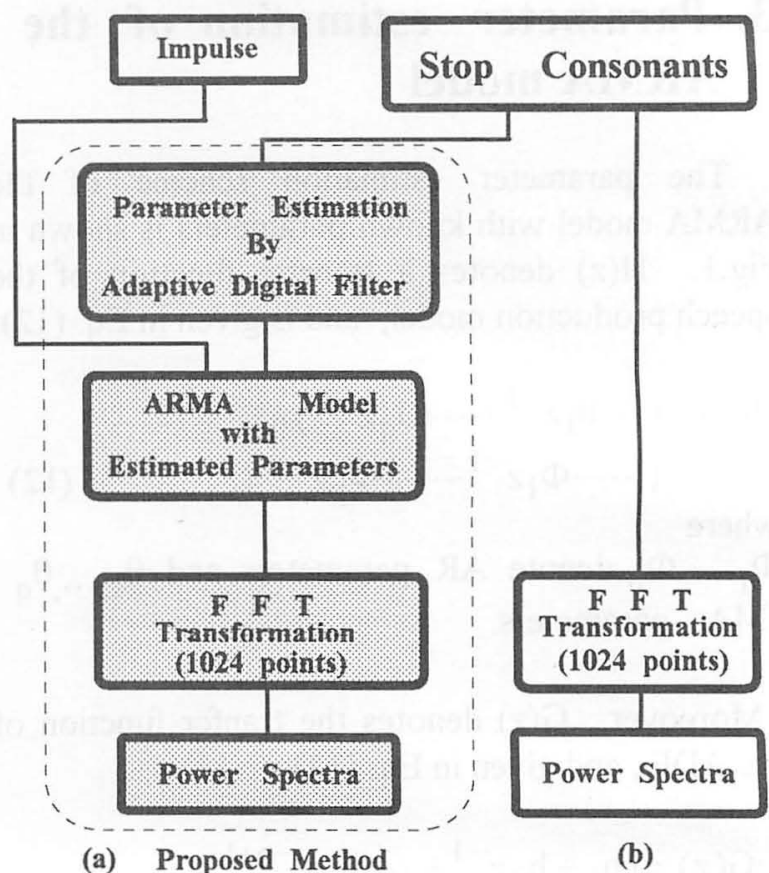

(a) Proposed Method

(b)

\section{Fig.5 Block diagram of parameter estimation (stop consonant)}

the above-mentioned procedures. The spectra of the voiced stop consonants are shown in Fig.7. In the both figures of Fig. 6 and Fig.7, the spectra based on the proposed method have the remarkable features.

\section{Conclusions}

In this paper, we proposed a fast parameter estimation method using the adaptive digital filter (ADF) based on the ASA method. The method is able to estimate the parameters of a short duration data such as $2.5 \mathrm{mS}$ long. As the applications of the proposed method, we presented two examples of the parameter estimation of the ARMA model and the natural stop consonants The results showed that the proposed method was useful for the parameter estimation of a short duration data. For further study, analysis of other phonemes and the words utilizing this method shall be included. We also intend to accomplish the recognition experiments of the stop consonants using a neural network. 

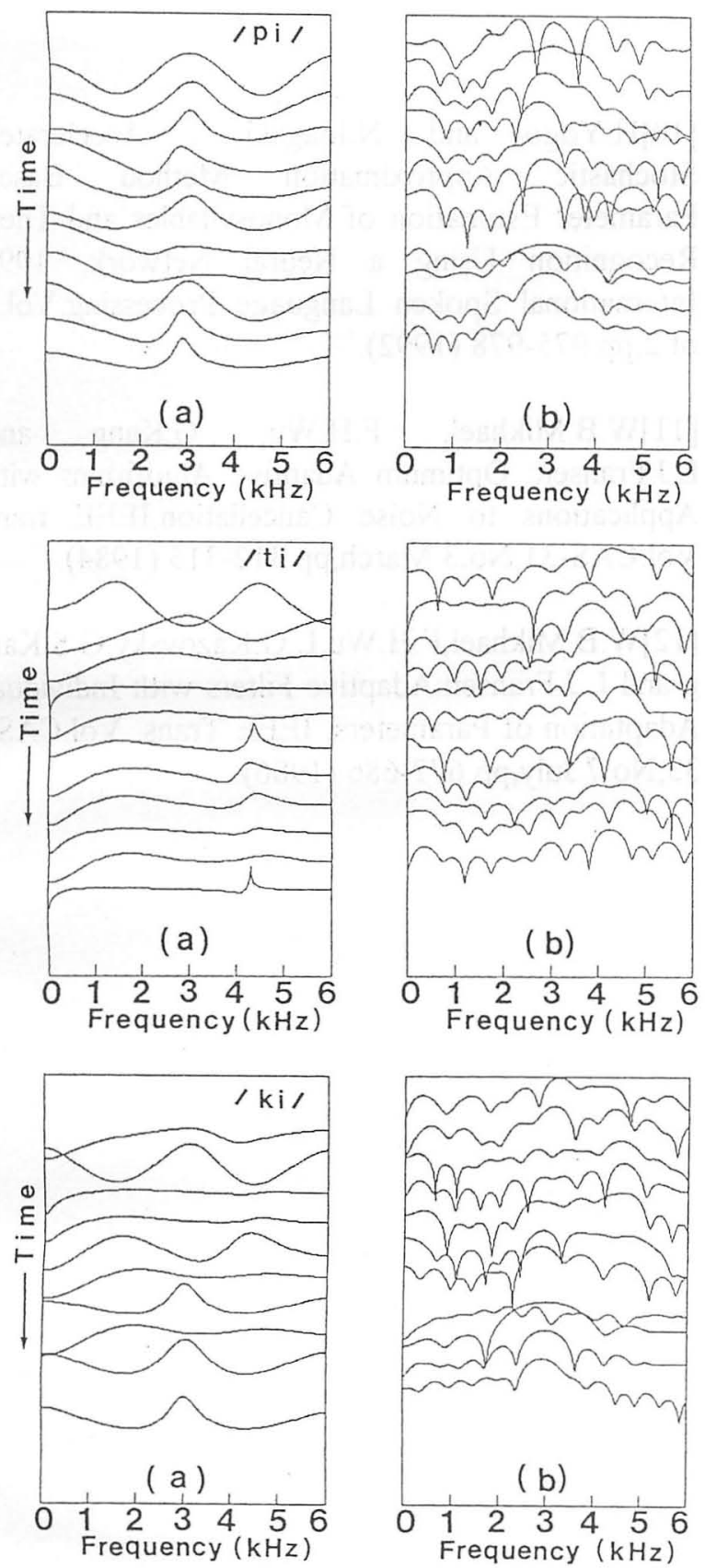

(a)

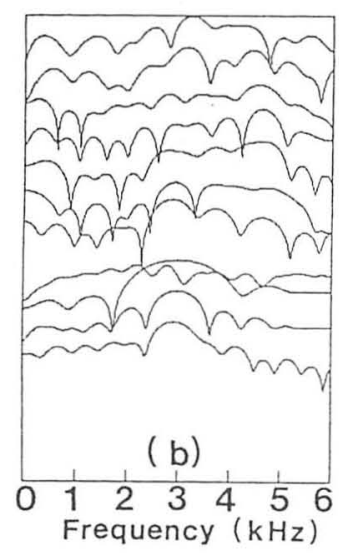

(b)
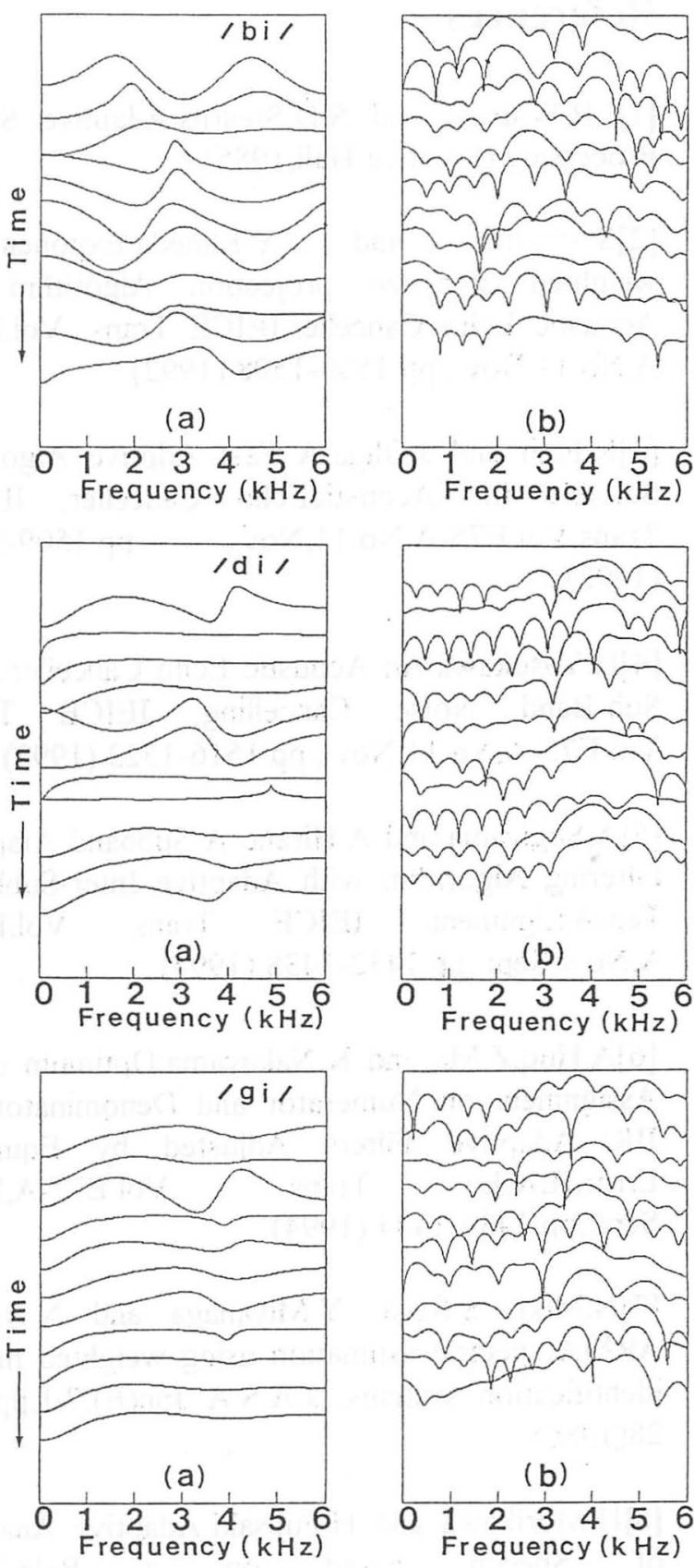

(a)

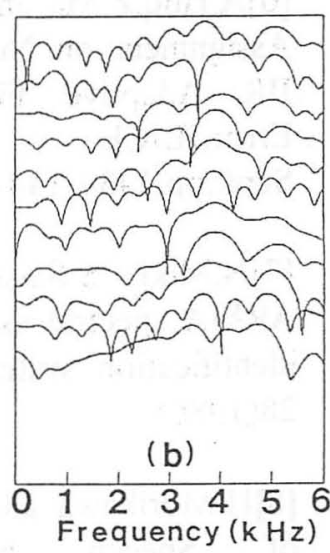

(b)
Fig.6 Spectra of the stop consonant (Unvoiced)

(a) Proposed method

(b) Conventional FFT method
Fig.7 Spectra of the stop consonant (Voiced)
(a) Proposed method
(b) Conventional FFT method 


\section{References}

[1] B.Widrow and S.D.Stearns:Adaptive Signal Processing (Prentice-Hall, 1985).

[2]S.Makino and Y.Kaneda:Exponentially weighted step-size projection Algorithm for Acoustic Echo Canceller,IEICE Trans. Vol.E75A,No.11,Nov., pp.1500-1508 (1992).

[3]K.Fujii and J.Ohga:A Fast Adptive Algoritjm Suitable for AcousticEcho Canceller, IEICE Trans.Vol.E75-A,No.11,Nov., pp.1509-1515 (1992).

[4]H.Yasukawa:An Acoustic Echo Canceller with Sub-Band Noise Cancelling, IEICE Trans. Vol.E75-A,No.11,Nov., pp.1516-1523 (1992).

[5]A.Sugiyama and A.Hirano:A Subband Afaptive Filtering Algorithm with Adaptive Inter-Subband Tap-Assignment, IEICE Trans. Vol.E77A,No.9,Sept.,pp.1432-1438 (1994).

[6]A.Huq,Z.Ma and K.Nakayama:Optimum order Assignment on Numerator and Denominator for IIR Adaptive Filters Adjusted by Equation Error,IEICE Trans. Vol.E77-A,No.9 Sept.,pp.1439-1444 (1994).

[7]N.Miki, S.Saga, Y.Miyanaga and N.Nagai: ARMA spectral estimation using weighted model identification systems. J.A.S.A Jpn(E),7,1,pp.2128(1986).

[8]H.Morikawa and H.Fujisaki:Adaptive Analysis of Speech based on a Pole-Zero Representation,IEEE Trans.ASSP,Vol.ASSP30,No.1,pp.77-88 (1982).

[9]A.Kikuchi,S.Ohmatsu and T.Soeda:Application of Adaptive Digital Filtering to the Data Processing for hte Environment System,IEEE Trans. ASSP-27,6,pp.790-803 (1979).
[10]H.Yogo and N.Inagaki : Accelerated Stochastic Approximation Method Based Parameter Estimation of Monosyllables and Their Recognition Using a Neural Network, 1992 International Spoken Language Processing, Vol.2 of 2,pp.975-978 (1992).

[11]W.B.Mikhael, F.H.Wu, G.Kang and L.J.Fransen: Optimum Adaptive Algorithms with Applications to Noise Cancellation,IEEE trans. Vol.CAS-31,No.3 March,pp.312-315 (1984).

[12]W.B.Mikhael,F.H.Wu,L.G.Kazovsky, G.S.Kan $\mathrm{g}$ and L.J.Fransen:Adaptive Filters with Individual Adaptation of Parameters, IEEE Trans. Vol.CAS33,No.7 July,pp.677-686 (1986). 